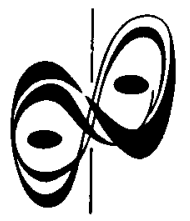

Acta Genet Med Gemellol 43: 124 (1994)

(C) 1994 by The Mendel Institute

International Congress

40th Anniversary of The Mendel Institute

\title{
Popliteal Aneurysm in Middle-Aged Identical Twins
}

\author{
G. Vigliotti ${ }^{1}$, E. Franco ${ }^{1}$, G. Cimino', P. Valitutti ${ }^{1}$, R. Russo², P. Angrisani², \\ M. Stabile ${ }^{3}$, A. Munno ${ }^{3}$ \\ Institutes of ${ }^{1}$ Vascular Surgery, ${ }^{2}$ Anatomical Pathology, Regional Hospital, Salerno; \\ ${ }^{3}$ Department of Genetics, "Cardarelli" Hospital, Naples, Italy
}

\begin{abstract}
Familial aggregation of isolated abdominal aortic and isolated intracranial aneurysms have been described. Therefore, districtual aneurysm without a systemic genetic disease (e.g. Marfan, Ehlers Danlos etc.) can have a genetic basis. The authors are reporting on the development, in the sixth decade of life, of popliteal aneurysm in two identical twins E.U. and E.C. Zygosity was established on the basis of blood-group determinations, HLA haplotypes and cytogenetic variants. Mild clinical symptoms (intermittent claudication, calf cramps) had begun in E.C. at 21 years of age. Later, at 52 years of age, a thrombosis of the aneurysmatic sac on one side required surgical treatment. E.U. showed no clinical symptoms until the age of 60 , when, within the interval of a few months, bilateral thrombosis had developed. The weight difference in the two subjects (E.C. $72 \mathrm{Kg}$, E.U. $64 \mathrm{Kg}$ ) may explain the difference between them as regards the age of onset of symptoms and body sides (one or both) affected. Angiographic, macroscopic and histologic aspects are documented. On surgical intervention, the aneursym was found at the biforcation of the popliteal artery, but no anomalous insertion of gastrocnemii appeared to cause pressure on the artery leading to aneurysmatic dilatation. The aneurysmatic sac measured $2.5 \mathrm{~cm}$ in diameter and on histological examination, using haematoxylin-eosin and Van Gieson stain, showed fragmented elastic, collagen fibres.
\end{abstract}

(Poster presentation)

Correspondence: Dr. M. Stabile, Department of Genetics, “Cardarelli ”' Hospital, 80131, Naples, Italy. 